\title{
Merits and Pitfalls of Multimodality Brain Monitoring
}

\author{
Jennifer Diedler $\cdot$ Marek Czosnyka
}

Published online: 14 April 2010

(C) Springer Science+Business Media, LLC 2010

In 1841 Magendi pioneered to measure cerebrospinal fluid pressure using the "sphygmometer," an instrument originally designed for assessment of blood pressure. From then on, it took 110 years before the first intracranial pressure (ICP) monitoring data has been published in 1951, first ever introducing the important key-word of "monitoring" in the context of the human brain [1]. From this moment the field of brain monitoring has been growing continuously. Apart from recording of ICP and cerebral perfusion pressure (CPP), modern multimodality brain monitoring comprises several techniques including assessment of brain tissue oxygenation $\left(\mathrm{P}_{\mathrm{br}} \mathrm{O}_{2}\right)$, brain metabolism, and cerebral blood flow. Noninvasive approaches are transcranial Doppler (TCD) ultrasonography, EEG monitoring, and near-infrared spectroscopy (NIRS).

Most of the experience in multimodality brain monitoring has been gained from comatose patients after traumatic brain injury, poor grade subarachnoid hemorrhage, or severe stroke. The main objectives of brain monitoring are (1) the detection of ongoing secondary brain injury following the primary insult, (2) guiding therapeutic interventions, and (3) prediction the outcome. Ideally, brain monitoring should prompt a timely and appropriate intervention and should be included in scheduled management protocols.

J. Diedler $(\bowtie)$

Department of Neurology, University of Heidelberg,

Heidelberg, Germany

e-mail: jennifer.diedler@med.uni-heidelberg.de

M. Czosnyka

Department of Clinical Neurosciences, University of Cambridge, Cambridge, UK

\section{Modalities and Their Merits}

1. Being a robust and only moderately invasive measure, ICP is probably the most commonly monitored parameter in neurocritical care. Although tissue shifts in the hemisphere contralateral to the sensor might be detected with a certain latency, it is usually regarded as a global measure. Some noninvasive methods to estimate ICP are available, but not yet introduced into clinical routine. ICP monitoring allows online detection of deterioration of intracranial pathology, such as freshly evolving mass lesions or development of brain edema. Effect of therapies can be monitored instantaneously. A close link between ICP and outcome has been established for different pathologies [2,3]. A number of secondary indices may be calculated from ICP and plotted as variables changing in time, the most common being CPP. Other indices are calculated based on various techniques of waveform analysis, such as compensatory reserve, or can be used to estimate cerebral autoregulation (pressure reactivity index, PRx) [4].

2. There are several other ways to assess cerebral autoregulation using tissue oxygenation or data from noninvasive devices such as NIRS or TCD to calculate different indices. Autoregulation indices have been shown to be good predictors of outcome in brain injured patients and allow "optimization" of global management variables like CPP or arterial blood pressure.

3. Cerebral blood flow can be monitored invasively using thermodilution probes or estimated noninvasively by TCD. Monitoring blood flow velocity over longer periods via TCD is challenging for the need to keep the probes in an accurate position. However, TCD has the 
great advantage that the operator can immediately assess the quality of the measurement, which may not be as straightforward with other monitoring modalities.

4. Changes in brain biochemistry can be assessed by microdialysis, requiring placement of a small catheter within the brain tissue. It allows analysis of various substances derived from the extracellular brain tissue such as glucose and its metabolites, lactate and pyruvate the neurotransmitter glutamate, and glycerol that is set free from membrane phospholipids during neuronal cell decay. Traditionally, the lactate/pyruvate ratio is interpreted as a sensitive indicator for ischemia. Likewise, moderately elevated glutamate levels may indicate ischemia in a reversible state, while excessively high levels and a concomitant increase in glycerol have been associated with an irreversible loss of neurons. However, there is still uncertainty about the exact implications and "critical thresholds" of the different parameters [5]; also probe location plays a crucial role.

5. Brain tissue oxygenation $\left(\mathrm{P}_{\mathrm{br}} \mathrm{O}_{2}\right)$ is measured by a microprobe that is commonly placed within the frontal white matter or the region of interest such as the penumbra of an infarct or adjacent to a hematoma. For TBI patients a link between low $\mathrm{P}_{\mathrm{br}} \mathrm{O}_{2}$ values and mortality has been established. However, if a tissueoxygen guided therapy on the other hand results in better outcome is still debated controversially.

6. Near-infrared spectroscopy (NIRS) can be used to assess regional cerebral tissue oxygenation noninvasively. Parameters provided by the NIRS, depending on the monitor model, are the amount of oxygenated and deoxygenated hemoglobin, the total tissue hemoglobin content that can be used to estimate local blood volume and the tissue oxygenation index, which may serve as a surrogate parameter for cerebral blood flow. Potential drawbacks of the pioneering technology are the influence of extracranial tissue confounding the NIRS data (recently reported as "solved" by spatialresolved spectroscopy), the mixing of arterial, capillary and venous compartments as well as the fact that brain swelling and hematomas can affect the accuracy and reliability of the NIRS parameters.

7. With the advent of digital EEG and quantitative analysis, continuous EEG monitoring has become more user-friendly. Indications include detection of (nonconvulsive) seizures, monitoring burst-suppression during barbiturate therapy, assessing levels of sedation, and prediction of outcome. Recent reports have shown the value of cEEG monitoring and quantitative EEG analysis to predict vasospasm in subarachnoid hemorrhage $(\mathrm{SAH})$ patients [6] or to detect relevant drops in cerebral perfusion in stroke patients. Electrocorticogram can be used to record cortical spreading depression, which are found in high incidence in all types of brain injury and have been linked to the occurrence of vasospasm. The exact implications of cortical spreading depression are yet to be demonstrated.

Despite each modality having its drawbacks, all are valuable tools to demonstrate working or failing brain physiology. If one modality fails to perform well (due to faulty transducers or placement in the "wrong region"), brain physiology usually can be explained based on information provided by others.

\section{Pitfalls}

1. One concept of multimodality brain monitoring is to detect and prevent evolving secondary brain injury before further irreversible damage occurs. This concept is based on the assumption that neurological outcome is determined by the primary injury, but also by a number of secondary insults, including development of cerebral edema or ischemia. However, a clear link between secondary brain damage and worse outcome has not been established for all diseases. Even in a seemingly straightforward relationship such as vasospasm in $\mathrm{SAH}$, randomized trials have failed to prove that prevention of vasospasm was associated with improved outcome [7].

2. The significance of adverse events detected by brain monitoring remains unknown. Do all monitored events, such as decreases of CPP or tissue oxygenation, metabolic crisis, hyperthermia or occurrence of spreading depolarization, contribute to poor outcome? Do they always require intervention-and if so, what are therapeutic options anyway? What thresholds should be applied? There is well documented evidence that individual thresholds might be required rather than generalized cut-offs. CPP thresholds for example will be different in a patient with intact versus disturbed autoregulation. The assumption that an already injured brain may poorly tolerate various kinds of systemic alterations may not hold in all circumstances [8]. The most important gap to be filled at the present stage is to establish a clear link from monitoring to management protocols. Proofs (in form of randomized trials) of a closed loop_- "patient-monitoring-doctor-protocoloutcome" - are to be delivered. Monitoring "per-se," in the absence of matching management protocols and effective therapies, cannot improve outcome.

3. It seems advisable to choose a multimodal approach in order to acquire a maximum of information (see Fig. 1). In some instances, "a little bit of brain 


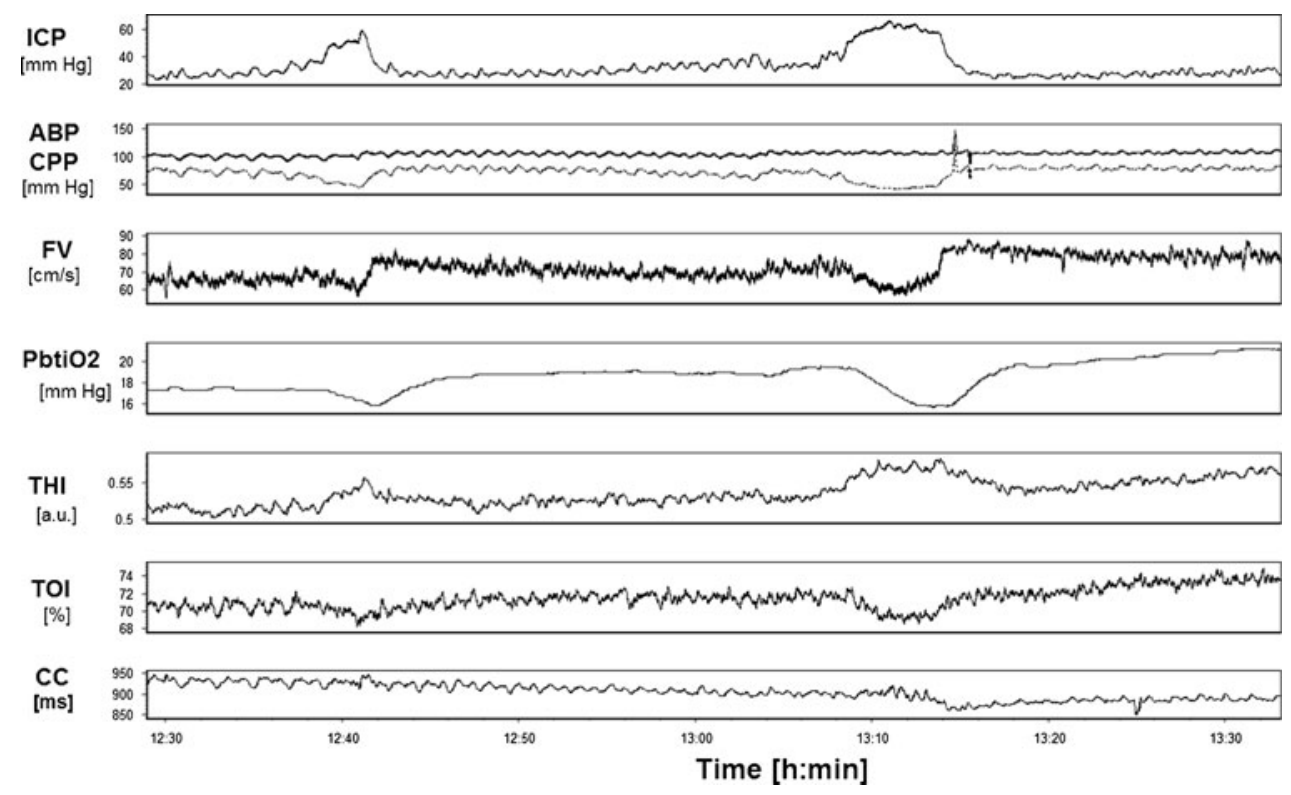

Fig. 1 Illustration of multimodality monitoring in a patient following $\mathrm{TBI}$, and showing secondary insults. $I C P$ intracranial pressure, $A B P$ peripheral blood pressure (upper) and $C P P$ cerebral perfusion pressure, $\mathrm{FV}$ blood flow velocity in $\mathrm{MCA}, \mathrm{PbtiO}_{2}$-tissue oxygen tension (Licox), THI total hemoglobin index (NIROS) being a dimensionless indicator of changes in cerebral blood volume, TOI

monitoring" may be more harmful than no monitoring at all. A single cerebral event can be caused by different pathophysiological mechanisms. An increase of ICP for example may be due to cerebral edema or transient hyperemia, each requiring different therapeutic responses. A therapeutic regimen, e.g., infusion of mannitol, based only on the information available from the ICP monitor, may even further aggravate the situation. While no protocols are available what modalities should be used, it seems wise to choose a multimodal approach. Furthermore, failures of sensors are common, the most frequent complication of brain monitoring was found to be device malfunction or dislodgement [9]. On the other hand, rates of hematoma or infections related to brain monitoring were reported between $3 \%$ and 5\% in a recent study.

4. In many modalities, probes pick up signals very locally. Probe placement therefore is crucial and it has to be kept in mind that thresholds for healthy and unhealthy brain tissue of course vary considerably.

5. Complexity of multimodal monitoring requires specialized and intelligent IT support. Various solutions (CMA Pilot, Edinburgh Browser, ICMPlus) are available and are becoming more popular nowadays. Not all neurocritical care units are ready to use them straightaway. total oxygenation index, mixed brain blood oxygen saturation (NIROS), $C C$ cardiac cycle. Two plateau waves were recorded. They were accompanied by a decrease in CPP, cerebral blood flow velocity and tissue oxygenation (note the typical "hyperemic" overshot after each wave), an increase in cerebral blood volume (rise in THI), and a decrease in brain blood saturation (fall in TOI).

6. Yet, the more complex the monitoring systems, the more the benefit from monitoring will depend on the skills of the clinicians to properly interpret and integrate the gathered information. While adequate training in interpretation seems indispensable, a proper evaluation of the interpretive ability of neurointensivists with respect to brain monitoring has not yet been undertaken.

7. Particularly, the missing link to outcome (commonly caused by a low number of studied cases) is a frequently raised criticism with respect to multimodality brain monitoring. Provided adequate clinicians' ability in data interpretation, a randomized trial on efficacy of brain monitoring, however, always will include testing the efficacy of a therapeutic regimen, employed as a result of the monitoring data. The benefit of monitoring thus will always be interwoven with the progress of therapies and development of new therapeutic standards. It is unrealistic to expect dramatic improvements in the outcome from monitors, especially when they are used to optimize existing therapies and no new therapies are employed [10].

In the study "Systemic glucose and brain energy metabolism after subarachnoid hemorrhage," Helbok et al. [11] provide a catchy example why monitoring of therapeutic effects of standard, seemingly trivial procedures such as 
titration of serum glucose may be required. They found that acute reductions in serum glucose, even within the normal ranges, were associated with brain energy metabolic crisis. Thus, a standard intervention, and for instance not alarmingly in the setting of a medical ICU, may severely compromise the acutely brain injured patient. While a link has been established between occurrence of metabolic crisis and outcome [12], this study, however, arises several questions: What are the underlying pathomechanisms? Is there a "metabolic penumbra" in other entities of brain injury? What glucose levels should be targeted for treatment? And ultimately, will an individualized glucose management as suggested by the authors, lead to improved outcome?

Disclosure ICMPlus is a brain monitoring software (www.medschl. cam.ac.uk/icmplus) licensed by Cambridge Enterprise Ltd, University of Cambridge, UK, and MC has a financial interest in a fraction of the licensing fee.

\section{References}

1. Guillaume J, Janny P. Continuous intracranial manometry. Rev Neurol. 1951;84:131-42.

2. Schwab S, Aschoff A, Spranger M, Albert F, Hacke W. The value of intracranial pressure monitoring in acute hemispheric stroke. Neurology. 1996;47:393-8.

3. Marmarou A, Anderson RL, Ward JD, Choi SC, Young HF, Eisenberg HM, et al. Impact of ICP instability and hypotension on outcome in patients with severe head trauma. J Neurosurg. 1991;75:S159-66.
4. Zweifel C, Lavinio A, Steiner LA, Radolovich D, Smielewski P, Timofeev I, Hiler M, Balestreri M, Kirkpatrick PJ, Pickard JD, Hutchinson P, Czosnyka M. Continuous monitoring of cerebrovascular pressure reactivity in patients with head injury. Neurosurg Focus. 2008;25:E2.

5. Schulz MK, Wang LP, Tange M, Bjerre P. Cerebral microdialysis monitoring: determination of normal and ischemic cerebral metabolisms in patients with aneurysmal subarachnoid hemorrhage. J Neurosurg. 2000;93:808-14.

6. Claassen J, Hirsch LJ, Kreiter KT, Du EY, Connolly ES, Emerson $\mathrm{RG}$, et al. Quantitative continuous EEG for detecting delayed cerebral ischemia in patients with poor-grade subarachnoid hemorrhage. Clin Neurophysiol. 2004;115:2699-710.

7. Macdonald RL, Kassell NF, Mayer S, Ruefenacht D, Schmiedek $\mathrm{P}$, Weidauer $\mathrm{S}$, et al. Clazosentan to overcome neurological ischemia and infarction occurring after subarachnoid hemorrhage (CONSCIOUS-1): randomized, double-blind, placebo-controlled phase 2 dose-finding trial. Stroke. 2008;39:3015-21.

8. Stocchetti N, Protti A, Lattuada M, Magnoni S, Longhi L, Ghisoni L, et al. Impact of pyrexia on neurochemistry and cerebral oxygenation after acute brain injury. J Neurol Neurosurg Psychiatry. 2005;76:1135-9.

9. Stuart RM, Schmidt M, Kurtz P, Waziri A, Helbok R, Mayer SA, et al. Intracranial multimodal monitoring for acute brain injury: a single institution review of current practices. Neurocrit Care. 2010 (Epub ahead of print).

10. Zygun D. Can we demonstrate the efficacy of monitoring? Eur $\mathbf{J}$ Anaesthesiol. 2008;25(Suppl 42):94-7.

11. Helbok R, Schmidt JM, Kurtz P, Hanafy KA, Fernandez L, Stuart $\mathrm{RM}$, et al. Systemic glucose and brain energy metabolism after subarachnoid hemorrhage. Neurocrit Care. 2010 (Epub ahead of print).

12. Sarrafzadeh A, Haux D, Küchler I, Lanksch WR, Unterberg AW. Poor-grade aneurysmal subarachnoid hemorrhage: relationship of cerebral metabolism to outcome. J Neurosurg. 2004;100:400-6. 